\title{
Creating Probabilistic Idiographic Performance Profiles from Discrete Feelings: Combining the IZOF and IAPZ models
}

\author{
M. Ryan FLET'T ${ }^{1}$
}

The purpose of the study was to determine if probabilistic profiling could be used to develop probabilistic individual zones of optimal functioning $(p I Z O F)$ profiles comprised from discrete feelings aggregated into valence-function categories (Hanin, 2000a). Ten male college tennis players developed individualized profiles, and then assessed their personal profile's items during changeovers of intrasquad matches. Support for the viability of using ordinal logistic regression (OLR; a foundation of the IAPZ method) to develop idiographic discrete performance-feeling profiles (common to the IZOF framework) is provided. Evidence of the idiosyncratic nature of the profiles and for the effectiveness of the IZOF-IAPZ integration is provided through visual assessments of figures, qualitative comparisons of feelingitems selected by each player, and statistical analyses of differences in the size (variance) and location (means) of zones - which revealed that 62 to 76 percent of zones were individually unique. The results of this study augment the IZOF model by (1) establishing that OLR can be used to develop discrete probabilistic (pIZOF) profiles, and (2) providing a variety of qualitative and quantitative methods of assessing idiosyncrasy.

Keywords: emotions, performance, tennis, ordinal logistic regression

\footnotetext{
${ }^{1}$ Department of Coaching and Teaching Studies, West Virginia University, Morgantown, WV, USA
} 
Tears, screams, and laughter are as much a part of sport as scoring and rules. Motivation, personal challenge, physical and psychological threat, reward opportunities, and the need for strong coping skills are implicit to both sport and emotion (Botterill \& Brown, 2002; Lazarus, 1991, 2000). If athletes have an interest in the outcome of their performance and training, they will experience emotions, and those emotions will likely influence their performance. This study explores the use of ordinal logistic regression (see, Kamata, Tenenbaum, \& Hanin, 2002) to develop probabilistic pIZOF profiles comprised of aggregates of discrete feeling states.

\section{Affect- and Feeling-Related Terminologies}

Although emotions are commonly experienced, the construct is complex and difficult to define. Emotions are functional processes that promote adaptational responses to environmental demands, and in turn, facilitate survival (Ekkikakis \& Petruzzello, 2000). Emotions are subjective in nature, involve physiological responses, and result in behavioral effects (Botterill \& Brown, 2002). As examples of what are considered to be emotions, Lazarus (2000) has identified 15 core discrete emotions, including: anger, anxiety, fright, guilt, shame, sadness, envy, jealousy, happiness, pride, relief, hope, love, gratitude, and compassion.

Clifford Saron elaborated in Goleman (1997, pp. 82-83): “Our commonsense vocabulary is a central problem in talking about emotions... doubt is not considered a basic emotion, like anger, happiness, or sadness. However, it is certainly a feeling state that we can identify." Feeling states describe a broad category that include both affective conditions and physiological sensations such as pain. General psychobiosocial (PBS) volitional and bodily form-states such as light, strong, quick, confident and fierce would be considered feeling states, rather than emotions. Such states are important for athletes, and are included on the positive-negative affect scale (PNA-77; Hanin, 1993; Syrja \& Hanin, 1997), but are not viewed as true emotions by researchers. The PNA has been used in scores of emotion and IZOF studies, and by releasing some constraints of true emotions, Hanin has developed a more complete list of performance-related PBS states. To account for the breadth of the PNA, the terms "feelings" or "feeling states" are used to encompass emotions, somatic sensations, and related states; also the term "feeling-performance profiles" describe the idiographic relationships between feeling states and performance.

\section{The IZOF and IAPZ Models}

Hanin's (1997, 2000a, 2003, 2007) IZOF model accounts for individual differences in emotion-performance relationships. The IZOF model also 
holds that athletes have zones of emotional intensity associated with optimal and dysfunctional performance; and that the size and locations of zones vary between individuals. In addition to driving a paradigm shift from nomothetic to idiosyncratic feeling-performance models, Hanin's work has influenced how we understand feelings. The IZOF construct has promoted the study of various emotions and feeling states (rather than anxiety-performance relationships alone); and the view that emotions are not inherently bad, but can be functional and adaptive (Botterill \& Brown, 2002; Hanin, 2000).

Despite these principal contributions, the IZOF model has limitations. IZOF-based analyses typically produce profiles with two zones that describe the feeling intensity associated with best and worst performance levels - referred to as the "in-out of the zone" principle (Hanin, 2000, p. 82). Though the IZOF model describes performance outcomes as probabilistic rather than absolute, studies rarely estimate the likelihood of each performance outcome across feeling intensity. Furthermore, the model does not account for moderate performances, undefined gaps between good- and poor-zones, and overlaps between zones (where both good and poor performance zones overlap at a certain level of intensity). The objective of this study and of the pIZOF method (as one method in the array of IZOF-based methodologies) is to generate profiles that account for moderate performance levels, and describe the exact probability of poor, moderate, and good performance levels.

OLR (also referred to as Logistic Ordinal Regression) is a statistical procedure that estimates the probability of ordinal outcomes such as poor, average, and good performance levels. For the purpose of individualized performance profiling, OLR is used to estimate the probability of performance levels across a range of feeling intensity (Kamata et al., 2002; Tenebaum, Kamata, \& Hanin, 2002). OLR was introduced to the feeling-performance profiling literature by Kamata et al., and became the foundation for the individual affect-related performance zones (IAPZ) method. Though every study that has applied Kamata's probabilistic method has measured general dimensional affect rather than discrete feelings, OLR is not exclusive to the IAPZ method or to dimensional affect: OLR could be applied to discrete feelings in the IZOF model.

IAPZs are defined by Johnson, Edmonds, Moraes, Medeiros Filho, and Tenenbaum (2007, p. 317) "as that range of affective intensity or [heart rate] within which an individual has the highest probability of performing at a specific performance level (e.g., poorly, moderately, or optimally)." Tenenbaum, Edmonds, and Eccles (2008, p. S15) elaborated: “...[IAPZs consist] of the reciprocal relationship between the perceived intensity of an affective state and the quality of an ensuing performance." The IAPZ method is, therefore, a 
process by which the idiosyncratic relationship between performance and the intensity of affect can be known and represented visually (see Figure 2 from these Results for an example, as this type of figure can be used for both the IAPZ and pIZOF types of probabilistic profiles). IAPZ profiles replace the all-or-none appearance of IZOF graphs with probabilistic curves for each performance level, and have contributed to the field by obtaining direct assessments of affect during performances (see, Cohen, Tenebaum, \& English, 2006; Edmonds, Mann, Tenenbaum, \& Janelle, 2006; Johnson, Edmonds, Moraes, et al., 2007; Medeiros Filho, Moraes, \& Tenenbaum, 2008).

Johnson, Edmonds, Kamata, and Tenebaum (2009) describe an eight-step procedure for developing IAPZs. Rather than assessing discrete feelings like anger or relaxation (which is common to the IZOF method), IAPZ studies have exclusively measured general dimensional affect, such as valence, arousal, and occasionally the functionality of each athlete's global affect state. This view of affect has empirical support (Russell, 1980, 1997; Russell, Weiss, \& Mendelsohn, 1989; Watson, 2002), but there are also disadvantages with the approach (see Lazarus, 1991, 2001). Researchers have assessed the IAPZ within the framework of Russell's (1980) circumplex model and corresponding use of the affect grid to measure feeling states. The affect grid (Russell et al.) is a 9X9, graph with two axes representing arousal (sleepiness to bighly aroused) and pleasure (displeasure to bighly pleasurable). The narrowing of focus - from feeling states and emotions to the affect sub-component - is motivated by the authors' efforts to achieve "parsimony and idiosyncratic generalizability" (Johnson et al., 2007, p.318). Though its parsimony makes the affect grid convenient for field research, it does not provide a comprehensive representation of emotion. Lazarus (1991, p. 59-60) explained the difference:

Conceiving of emotions as discrete categories, each of which can be placed on a dimension from weak to strong, is very different from thinking of them as overlapping dimensions, in which many categories are reduced to a few and their distinct qualities lost or blurred.

Watson (2002) recommended that global dimensions of affect be assessed in combination with assessments of specific affect categories (e.g. discrete feelings). This approach avoids the conflict between the dimensional versus discrete approaches, and allows researchers to capture more information. Consider the following example: A person could perceive sadness and anger as being equally unpleasant, and thus give them the same valence score. Each feeling might also be considered equally helpful, or functional. As such, measuring only valence and functionality would not distinguish between the two distinct feelings. Despite citing Watson's work, IAPZ studies do not provide multilevel 
representation of emotions. Hanin's (2000a) PNA-based method of assessing IZOF meets Watson's recommendations because discrete emotions can also be categorized by their valence and functionality (i.e., positive-functional, positivedysfunctional, negative-functional, and negative-dysfunctional categories; herein PF, PD, NF, and ND respectively).

The probabilistic method developed by Kamata et al. (2002) has been applied to the IAPZ method, but has not been used to profile discrete feelings that are characteristic of the IZOF model. Although there is no evidence that OLR cannot be used to profile discrete feelings, there is also no previous research indicating that the two are capable of producing individualized, probabilistic ("pIZOF") profiles. The purpose of this study is to determine, for the first time, if OLR can generate pIZOF profiles comprised of aggregates of discrete feelings. Just as IAPZ studies have used OLR to develop probabilistic profiles using dimensional affect, it is hypothesized that discrete feelings can be aggregated into valence-function categories and that pIZOF profiles for poor, average, and good performance levels can be developed for each category. Such pIZOF profiles would have the advantage of being comprised from discrete feeling states and/or aggregated valence-function categories. The integration of IZOF profiles and probabilistic analysis will be successful if individual profiles: have unique discrete feelings, appear unique to one another, look like IAPZ figures, and if the size and location of poor, average, and good zones vary across individuals. A secondary purpose of the study is to illustrate that the statistical use of OLR is not exclusive to IAPZ modeling; and in doing so, demonstrate that IAPZ research's limited use of OLR (limited to profiling dimensional affect) does not constitute a model that "replace[s] Hanin's (2000) concept of affectperformance linkage" (Golden, Tenenbaum, \& Kamata, 2004, p. 37).

\section{Method}

\section{Participants}

Ten male players from an NCAA Division I tennis team were recruited to participate in this study. On average, participants were 20.1 years of age $(S D=$ $1.66)$, had 13.7 years of tennis experience $(S D=2.58)$, and 2.5 years of college tennis experience $(S D=1.27)$. They had previous experience working with a sport psychology consultant, but not with the IZOF models or IAPZ. Because of these characteristics of the sample, it was expected that participants would reflect a high level of emotional self-awareness and tennis experience. According to Raglin and Hanin (2000), experienced, competitive athletes provide more reliable recall and emotional awareness than do novices - important attributes for idiographic profiling. 


\section{Instruments}

Individual optimal and dysfunctional performance zones are identified through stepwise procedures known as individualized emotion profiling (IEP; Hanin, 1997, 2000b, 2003), which are described in the procedure. The IEP uses the PNA-77 and Borg CR-10 instruments (described below). Individualized forms were developed to collect data during matches.

Positive-negative affect list. The positive and negative affect list (PNA-77; Hanin \& Syrja, 1995, 1996) was established by Hanin as part of his stepwise IEP procedure. The PNA is a list that describes athletes' feeling states during competition, and has mean intraindividual Cronbach alpha values ranging from .76-.90 (Hanin \& Syrja, 1996). With respect to hedonic tone, there are 40 positive (e.g., relaxed, confident, energetic, determined, quiet, satisfied) and 37 negative (e.g., intense, aggressive, tight, sluggish, afraid, angry) items. Items on each row are synonyms, so participants are not permitted to choose multiple items from any row. In addition to the 77 items in the PNA, participants were urged to include their own feeling-adjectives.

Borg CR-10. Borg's category ratio scale (CR-10; Borg, 1998) has described the intensity of feelings in the IZOF model since its revision (Hanin, 1997). The CR-10 was modified by Hanin and colleagues (Hanin \& Syrja, 1995; Tummavuori \& Hanin, 2000), and the reliability of the CR-10 in IZOF research was established by Hanin and Syrja (1996). In a later study of 200 athletes' 46,934 intensity ratings, the distribution of verbal anchors along the intensity continuum were confirmed to be appropriate (Tummavuori \& Hanin, 2000). The CR-10 is a 12-point scale from 0 to 11 . The descriptive anchors for each level on the continuum are described in Figure 1.

Monitoring form. The first section of the monitoring form (see Figure 1) identified the context (service or return), outcome of the game (won / lost), and score in the set. Next, perceptions of performance were assessed across an 11-point Likert scales (from 0-10). The descriptive words "poor," "average," and "good" were used to anchor the Likert scale at 0, 5, and 10 respectively. The third section included the feeling states and intensity Likert scales. 


\begin{tabular}{|c|c|c|c|c|c|c|c|}
\hline Type of Game: Serve / Retum & Won & $1 / \mathrm{L}$ & ost & Set Score: & & $\overline{\text { you }}$ & $-\overline{\text { him }}$ \\
\hline \multicolumn{8}{|l|}{ Rate your feelings about... } \\
\hline My performance was: & Poor & & & Average & & & Good \\
\hline Performance: & 01 & 2 & 3 & $\begin{array}{lll}4 & 5 & 6\end{array}$ & 7 & 8 & $9 \quad 10$ \\
\hline The challenge of the game (task) was: & Poor & & & Average & & & Good \\
\hline Challenge: & $0 \quad 1$ & 2 & 3 & $\begin{array}{lll}4 & 5 & 6\end{array}$ & 7 & 8 & $9 \quad 10$ \\
\hline $\begin{array}{l}\text { My skills to meet my goals for the game } \\
\text { were: }\end{array}$ & Poor & & & Average & & & Good \\
\hline Skill: & $0 \quad 1$ & 2 & 3 & $4 \quad 5 \quad 6$ & 7 & 8 & $9 \quad 10$ \\
\hline
\end{tabular}

Emotional Intensity

\begin{tabular}{|c|c|c|c|c|c|c|c|c|c|c|c|c|c|c|c|c|}
\hline \multirow{2}{*}{\multicolumn{2}{|c|}{ 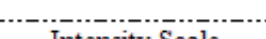 }} & \multirow{3}{*}{$\begin{array}{l}(\mathrm{P}+) \\
(\mathrm{P}+)\end{array}$} & \multirow[b]{2}{*}{ Energetic } & \\
\hline & & & & 0 & .5 & 1 & 2 & 3 & 4 & 5 & 6 & 7 & 8 & 9 & 10 & $11^{*}$ \\
\hline 0 & nothing at all & & Confident & 0 & .5 & 1 & 2 & 3 & 4 & 5 & 6 & 7 & 8 & 9 & 10 & $11^{*}$ \\
\hline 0.5 & very very little & $(\mathrm{P}+)$ & Determined & 0 & .5 & 1 & 2 & 3 & 4 & 5 & 6 & 7 & 8 & 9 & 10 & $11^{*}$ \\
\hline 1 & very little & $(\mathrm{P}+)$ & & 0 & .5 & 1 & 2 & 3 & 4 & 5 & 6 & 7 & 8 & 9 & 10 & $11^{*}$ \\
\hline 2 & little & $\mathrm{N}+)$ & Aggressive & 0 & 5 & & 0 & & 4 & 5 & 6 & 7 & 8 & 9 & 10 & \\
\hline \multirow{2}{*}{3} & \multirow{2}{*}{ moderate } & $\mathrm{N}+2$ & Intense & 0 & 5 & 1 & 2 & 3 & 4 & 5 & 6 & 7 & 8 & 9 & 10 & $11^{*}$ \\
\hline & & $(\mathrm{N}+)$ & & 0 & .5 & 1 & 2 & 3 & 4 & 5 & 6 & 7 & 8 & 9 & 10 & $11 *$ \\
\hline 5 & \multirow[t]{3}{*}{ much } & $(\mathrm{N}+)$ & & 0 & .5 & 1 & 2 & 3 & 4 & 5 & 6 & 7 & 8 & 9 & 10 & $11^{*}$ \\
\hline \multirow{2}{*}{6} & & & & & & & & & & & & & & & & \\
\hline & & (N-) & Annoyed & 0 & .5 & 1 & 2 & 3 & 4 & 5 & 6 & 7 & 8 & 9 & 10 & $11^{*}$ \\
\hline 7 & \multirow[t]{3}{*}{ very much } & (N-) & Discouraged & 0 & .5 & 1 & 2 & 3 & 4 & 5 & 6 & 7 & 8 & 9 & 10 & $11^{*}$ \\
\hline & & (N-) & Doubtful & 0 & .5 & 1 & 2 & 3 & 4 & 5 & 6 & 7 & 8 & 9 & 10 & $11 *$ \\
\hline 9 & & (N-) & Fearful & 0 & .5 & 1 & 2 & 3 & 4 & 5 & 6 & 7 & 8 & 9 & 10 & $11^{*}$ \\
\hline \multirow{2}{*}{10} & \multirow{2}{*}{$\begin{array}{l}\text { very very } \\
\text { much }\end{array}$} & & & & & & & & & & & & & & & \\
\hline & & (P-) & Quiet & 0 & .5 & 1 & 2 & 3 & 4 & 5 & 6 & 7 & 8 & 9 & 10 & $11 *$ \\
\hline \multirow{3}{*}{11 * } & maximal & (P-) & Satisfied & 0 & .5 & 1 & 2 & 3 & 4 & 5 & 6 & 7 & 8 & 9 & 10 & $11^{*}$ \\
\hline & possible & (P-) & Rapid & 0 & .5 & 1 & 2 & 3 & 4 & 5 & 6 & 7 & 8 & 9 & 10 & $11^{*}$ \\
\hline & & (P-) & & 0 & .5 & 1 & 2 & 3 & 4 & 5 & 6 & 7 & 8 & 9 & 10 & $11^{*}$ \\
\hline
\end{tabular}

Comments (Not necessary, but please include if there is something you would like to note):

Figure 1. Example of the Monitoring Form used by Participant \#2 (adopted from Hanin, 2000, p. 313) 


\section{Procedure}

The study was approved by the university's Institutional Review Board and described to the head coach for his approval before obtaining informed consent from players. The NCAA tennis season begins with an autumn preseason. In the first week of the preseason, players attended an orientation meeting where informed consent was obtained and individual emotion-performance profiles were developed. Participants completed a monitoring form during the changeovers of intrasquad matches. Each participant played between 5-8 $(M=$ 6.90; $S D=0.99)$ matches and completed 52-138 forms $(M=91.8 ; S D=23.40)$. An average of $13.3(S D=2.61)$ observations were recorded from each match. Player 6 had only 52 observations because he missed three matches due to an injury and then asked to be removed from the study due to frustration from under-performing. Poor-, average-, and good-intensity zones were determined based on the most-likely performance outcome at each point on the feelingintensity continuum for each valence-function category of feeling states (See, Medeiros Filho et al., 2008, p. 450).

Stepwise procedure. Participants identified 12-14 relevant feelings based on three best and three worst performances over the past two years (see Figure 1). In accordance with Hanin's (2000a, p. 162) recommendations for field research, players simplified their profiles to include only the 12 items that they felt were most important - including 2-4 items in each of the four valencefunction categories (i.e., PF, NF, PD, and ND). One participant had a 13-item profile. Next, the Borg CR-10 scale was used to estimate the functional and dysfunctional zones for each discrete content item. Participants competed the monitoring form during each changeover in every match, rather than merely reflecting on pre- or post-competition states after the match.

Data collection. After establishing and refining each IZOF profile and explaining the in vivo data collection procedures, the next stage of the procedure involved actually monitoring feelings and performances during matches. Participants played a round robin of matches against their teammates to determined rankings within the team (only the top six players play singles matches in the NCAA). In a post-study survey of players, the average rating of how competitive the challenge-matches were was 6.40 (on a scale from 1-10; $S D=2.31$ ). Six participants rated the competitiveness of the matches between 7-8. There were two outliers who rated competition as a 2-3 - neither player performed as well as he had hoped. The average rating of how important the matches were was $7.05(S D=1.32)$. One participant rated the importance of the matches uniquely lower than the rest of the team (a " 4 " out of 10). 
During each "changeover" (odd game), participants completed the monitoring form for the one game (of the two) that had the most "non-average" (e.g., very good or poor) performance. If both games prior to the changeover had extremely good or poor performances, players completed a form for both. All players were told to complete the forms quickly, so as not to over-think or distract from performance. In pilot testing and in this study, forms were completed in 25-40 seconds, and were considered to be minimally-moderately distracting. In a post-study survey of participants, the average rating of how distracting the forms were was 4.05 (on a scale from $1-10 ; S D=2.81$ ). Two players found the forms to be quite distracting, rating it as 7.5 and 9. The player who provided the latter rating withdrew from the study after five matches (Player 6, whose data was included in the analysis). All participants felt that they completed the forms very accurately $(M=8.2 ; S D=1.42)$. The primary investigator attended all intrasquad challenge-matches to ensure that forms were available and that participants' concerns could be addressed.

\section{Treatment of Data: Aggregating variables and OLRs}

Aggregated performance levels and feeling-categories. Participants rated their performances on a scale from $0-10$. By convention, these ratings were converted to ordinal data by defining ratings between 0-3 as poor performances, 4- 6 as average performances, and 7-10 as good performances. Participants were asked if these parameters for the three performance levels were agreeable (e.g., "do you consider a ' 3 ' to be poor? A ' 6 ' to be average? A ' 7 ' to be good? Etc." For two participants, the ordinal parameters were modified because they defined their performance levels differently in relation to the Likert scale, which initially resulted in extreme distributions of good performances. After consulting with each player, Player 1's parameter for good performance was adjusted to 6-10; and Player 3's parameters were set at 0-4, 5-7, and 8-10. Each of the 12-13 discrete feeling states identified by participants were grouped into one of four valence-function categories determined through the IEP process. Rather than conducting analyses for each discrete item for each player, analyses were conducted for the aggregated value of items in each category. In addition to simplifying each player's profile, it also made it possible to compare individuals because whereas few players selected the same discrete feeling-items, every player shared the same aggregated valence-function categories.

Parameter estimates, curves and zone parameters. In keeping with Kamata et al.'s (2002) method, poor and average performances were each split into highand low-intensity subgroups based on the mean feeling intensities for good performances. The resulting five subgroups acted as dependent measures in the OLR: poor-low, poor-high, average-low, average-high, and good. Parameter 
estimates from the OLRs conducted on the four feeling-categories for each participant generated probabilistic estimates of performance across feeling intensity. The estimates were then used to identify poor, average, and good intensity zones. In total, $40 \mathrm{pIZOF}$ graphs (representing the 4 feeling-categories and 10 participants) were generated with three to five performance curves in each (e.g., see Figure 2). The OLR spreadsheets that produced each graph were consulted to identify the exact parameters of each intensity zone. This process was repeated for each intensity zone of the 40 pIZOF graphs and summarized in Figure 3, which is a type of figure used by Medeiros Filho et al. (2008) to summarize performance probability curves.

\section{Results}

Of the 918 observations in this study, seven forms were excluded due to incomplete data or because ratings for every item were identified with a single circle (out of frustration). Three methods were used to validate the pIZOF method and to assess interindividual differences, including: qualitative comparisons of the items players selected; visual inspection of individual graphs; and statistical comparisons of the location (means) and size (variances) of each zone.

\section{Qualitative differences in individual profile items}

One of the most idiosyncratic elements of feeling-performance profiles was the choice of discrete feeling states. Though participants shared four common feeling-categories (e.g., PF, etc.), the exact items in each category were self-selected and unique across participants. Before comparing individual item selections, synonymous items (e.g. angry and furious) on each row of the PNA-77 (Syrja \& Hanin, 1997) grouped together - which resulted in a more conservative comparison. The distribution of choices presented in Table 1 supports the notion of idiosyncratic differences in players' selections. Six rows from the positive list and eight rows from the negative list were supported by the majority of players (five or more). The satisfied, confident, aggressive, and irritated rows received the strongest support (7-8 players). However, all 28 rows of items were supported by at least one player. Idiosyncratic variability was even greater when comparing exact discrete items, rather than synonymous rows. 
Table 1

Player Support for each Row of (Synonymous) Positive and Negative Content Item Descriptors

\begin{tabular}{lcll}
\hline \multicolumn{1}{c}{ Positive Items } & N & \multicolumn{1}{c}{ Negative Items } & N \\
\hline glad, pleased, satisfied, contented & 8 & angry, aggressive, furious, violent & 7 \\
confident, certain, sure & 7 & annoyed, irritated, distressed & 7 \\
relaxed, comfortable, easy & 6 & afraid, fearful, scared, panicky & 6 \\
determined, set, settled, resolute & 6 & discouraged, dispirited, depressed & 6 \\
quick, rapid, fast, alert & 6 & doubtful, uncertain, indecisive, irresolute & 6 \\
lighthearted, carefree & 5 & intense, fierce & 6 \\
active, dynamic, energetic, vigorous & 4 & jittery, nervous, uneasy, restless \\
calm, peaceful, unhurried, quiet & 4 & tense, strained, tight, rigid & 5 \\
delighted, overjoyed, exhilarated & 3 & anxious, apprehensive, worried & 5 \\
excited, thrilled & 3 & helpless, unsafe, insecure & 4 \\
nice, pleasant, agreeable & 2 & inactive, sluggish, lazy & 3 \\
brave, bold, daring, dashing & 2 & tired, weary, exhausted, worn out & 3 \\
inspired, motivated, stimulated & 2 & concerned, alarmed, disturbed, dissatisfied & 1 \\
cheerful, merry, happy & 1 & sorry, unhappy, regretful, sad, cheerless & 1 \\
\hline
\end{tabular}

Note: Maximum player support value was 10. Rows of synonymous items were arranged based on Hanin's PNA (2000).

\section{Visual differences in zone profile figures}

Figure 2 shows individual performance probability curves of across feeling intensity (i.e., a pIZOF profile) of the same kind published in previous IAPZ studies. These figures provide further evidence that the integration of probabilistic OLR and traditional IZOF profiling was successful, as the pIZOF profile graphs were typical of those from previous IAPZ studies. Individual profiles were simplified and presented for inter-individual comparison in Figure 3. 


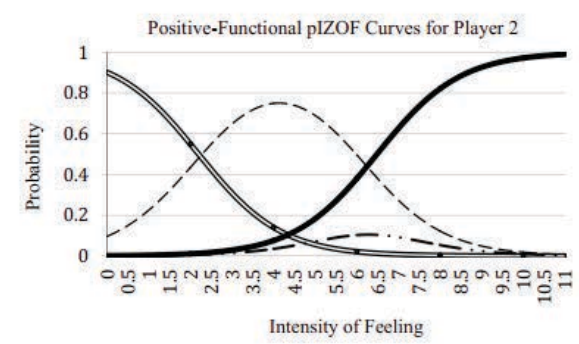

Negative-Functional pIZOF Curves for Player 2

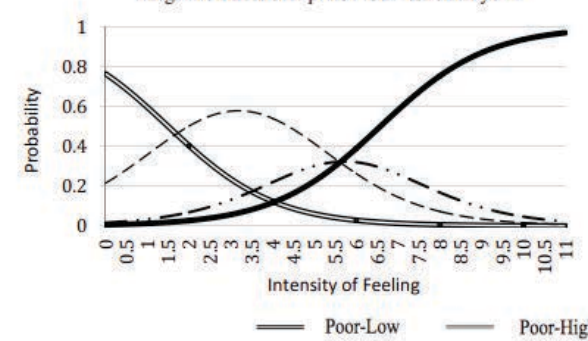

Positive-Dysfunctional pIZOF Curves for Player 2

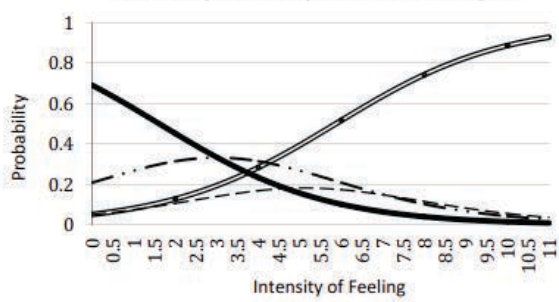

Negative-Dysfunctional pIZOF Curves for Player 2

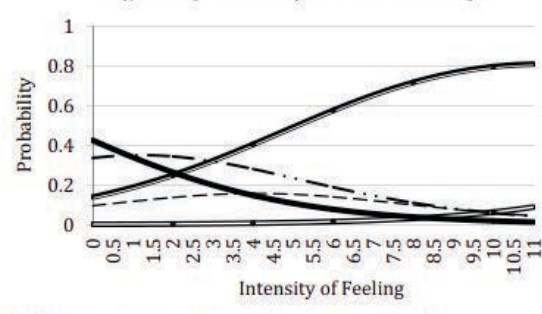

Figure 2. pIZOF profiles describing the probability of various performancelevels across feeling intensity. Profiles represent different valence-function categories for various participants. Curves within each profile represent five possible performance-levels: poor performances with low (Poor-Low) and high (Poor-High) intensity ratings; average performances with low (Ave-Low) and high (AveHigh) intensity ratings; and good performances (good). 

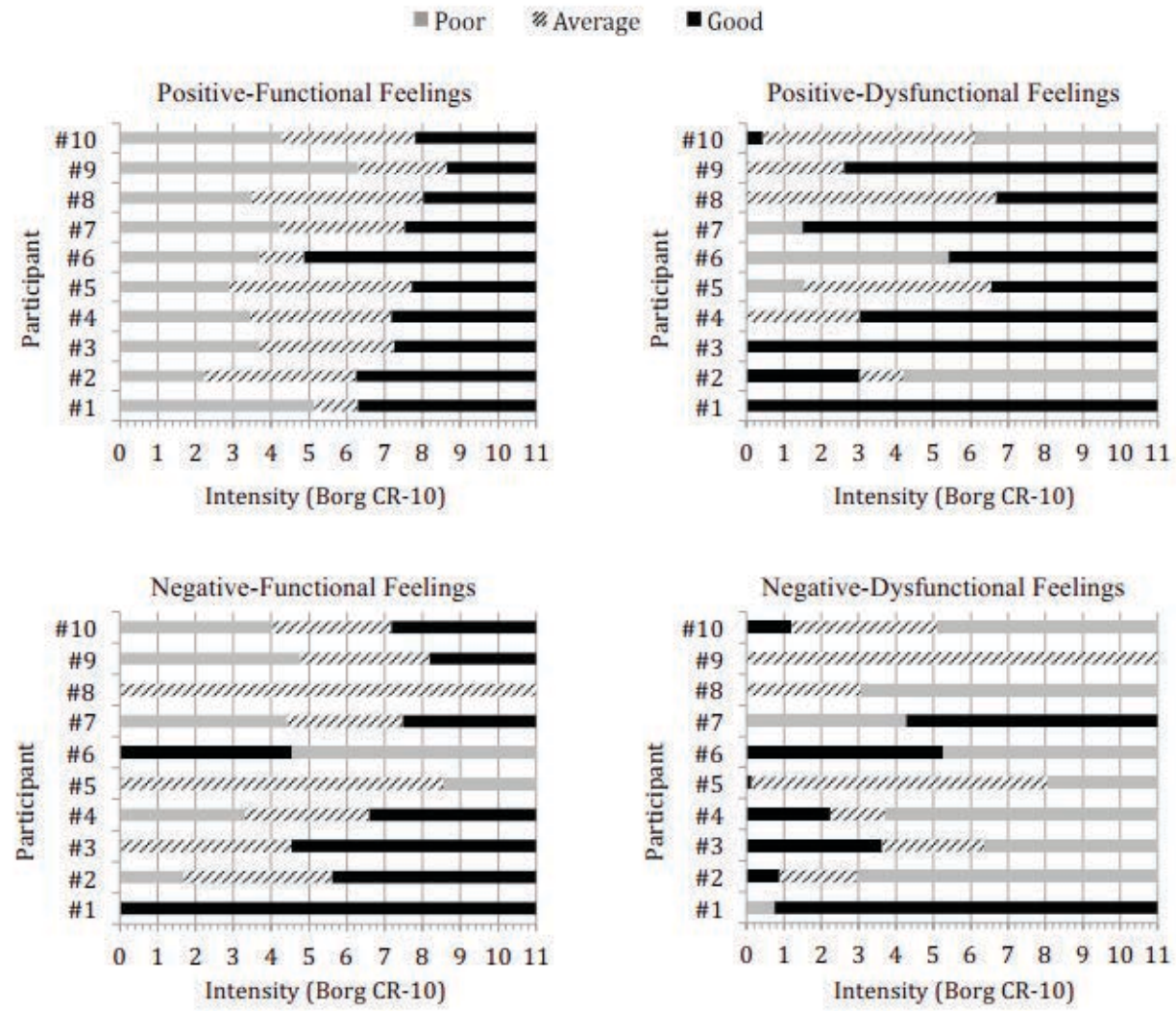

Figure 3. Performance zones for participants across intensity of each valencefunction category. Participants are displayed as rows assigned to the y-axis. The $\mathrm{x}$-axis represents the intensity of feelings (from 0-11 using the Borg CR-10). Good-performance zones are represented in black, poor-zones in dark grey, and average-zones with diagonal lines.

Graphic representation of probability curves. Figure 2 depicts the probability (from 0.0-1.0 on the Y-axis) of various performance levels across the full spectrum of feeling intensity (ranging from 0-11). Each performance level is represented by a different curved line within each graph. At any point of feeling intensity (on the $\mathrm{X}$-axis), the area under the curved performance-lines represents the probability of each line's performance level. If the probabilities for each performance-line at a fixed point on the $\mathrm{X}$-axis were added together, they would sum to exactly 1.0 (or a 100\% probability). For example, at 0 intensity of ND feelings, there are 
$.05, .05, .20$, and .70 probabilities for each of the four performance curves (i.e., a $70 \%$ chance of good performance if negative-dysfunctional feelings are absent).

Combined summary of individual curves. Each of the 10 participants had four different individual figures from the above section (one for each valence-function category, resulting in 40 total graphs). Rather than reporting all 40 graphs, each player's zones are summarized across the four valence-function categories in Figures 3. The zones within each player's profile are determined by the most likely performance level at any given point of intensity, based on the probability curves described in the previous section. These graphs provide additional evidence OLR was effectively applied to discrete IZOF feelings, and reaffirmed the notion of idiosyncratic emotion-performance relationships. Each graph in Figure 3 is described above.

The intensity zone profiles for PF feelings were identical in that all players went from poor to average to good performance levels as intensity increased. Beyond this pattern of results, individual profiles appear to differ in terms of the location and/or size of each zone - which supports the hypotheses. It is also worth noting that some players' profiles were quite similar: Player 3 and 4 were alike, as were Players 7, 10, and to some degree 5, and 8.

Intensity zones for NF feelings displayed more idiosyncratic patterns. Except for Players 5 and 6, performances improved as intensity increased. Players 5 and 6 are atypical because functional feelings normally improve performance - whether they are positive or negative in valence. Players 1 and 8 had only one intensity zone; and Players 3, 5, and 6 had only two zones. Players 7, 9, and 10's profiles were quite similar.

Intensity zones for ND feelings displayed even more idiosyncratic patterns. Player 9 had one zone. Players 1, 5, 6, 7, and 8 had two zones (Players 5's goodzone is so small that there were effectively two likely outcomes). Players 2, 3, 4, and 10 had three zones, and each of their profiles appears unique. Performance diminished as intensity increased for most participants - with the exceptions of Players 1 and 7.

The PD intensity zones had the most unique results. Player 1 and 3 had only one intensity zone. Players 4, 6, 7, 8, and 9 had only two zones. Players 2, 5, and 10 had three zones, and each of their profiles appears to be very different. What makes these results peculiar is that all but two players displayed improved performance with increased intensity of dysfunctional feelings. 


\section{Quantitative interindividual comparisons}

Verifying the idiographic nature of the profiles is important because it reinforces that the pIZOF system produces profiles that are consistent with the core of the IZOF model: idiosyncrasy. There are two ways that individual profiles can vary - in either the location or in the size of individual zones. Differences in the location of each zone were assessed using ANOVA's and $t$ tests, and the sizes of zones were assessed by comparing the zone-variances (see Table 2).

Table 2

Differences in Zone Location and Size Across Feeling-Category and Performance Level

\begin{tabular}{|c|c|c|c|c|}
\hline Performance Level & PF & NF & ND & PD \\
\hline \multicolumn{5}{|l|}{ Poor } \\
\hline ANOVA & $\begin{array}{c}F(9,230)= \\
36.57\end{array}$ & $\begin{array}{c}F(9,230)= \\
37.19\end{array}$ & $\begin{array}{c}F(9,230)= \\
33.16\end{array}$ & $\begin{array}{c}F(9,230)= \\
87.59\end{array}$ \\
\hline Significant $t$ tests (.001) & 16 & 21 & 13 & 20 \\
\hline Significant $t$ tests (.01) & 23 & 26 & 21 & 28 \\
\hline Levene's & 4.59 & 8.56 & 4.66 & 15.11 \\
\hline ignificant comparisons (.001) & 13 & 18 & 13 & 18 \\
\hline Significant comparisons (.01) & 16 & 24 & 17 & 25 \\
\hline \multicolumn{5}{|l|}{ Average } \\
\hline ANOVA & $\begin{array}{c}F(9,432)= \\
13.41\end{array}$ & $9,432)=\mathbf{3 8 . 0 1}$ & $\begin{array}{c}F(9,432)= \\
27.40\end{array}$ & $\begin{array}{c}F(9,432)= \\
45.22\end{array}$ \\
\hline Significant $t$ tests (.001) & 13 & 19 & 21 & 29 \\
\hline Significant $t$ tests (.01) & 19 & 24 & 27 & 34 \\
\hline Levene's & 6.95 & 11.84 & 10.07 & 15.83 \\
\hline Significant comparisons (.001) & 13 & 20 & 23 & 25 \\
\hline Significant comparisons (.01) & 20 & 25 & 25 & 33 \\
\hline \multicolumn{5}{|l|}{ Good } \\
\hline ANOVA & $\begin{array}{c}F(9,219)= \\
18.43\end{array}$ & $\begin{array}{c}F(9,219)= \\
\mathbf{6 0 . 9 6}\end{array}$ & $\begin{array}{c}F(9,218)= \\
33.55\end{array}$ & $\begin{array}{c}F(9,217)= \\
28.57\end{array}$ \\
\hline Significant $t$ tests (.001) & 9 & 20 & 20 & 18 \\
\hline Significant $t$ tests (.01) & 23 & 28 & 25 & 26 \\
\hline Levene’s & 10.43 & 18.33 & 6.41 & 9.17 \\
\hline Significant comparisons (.001) & 23 & 20 & 20 & 18 \\
\hline Significant comparisons (.01) & 31 & 27 & 24 & 24 \\
\hline
\end{tabular}

Note: Significant values at $p<.001$ are presented in bold font. Note that all ANOVAs and Levene's tests were significant at that level. The acronyms for the columns represent PF, NF, ND, and $\mathrm{PD}$ valence-function feeling-categories. 
Differences in location of zones. Twelve separate one-way ANOVAs were conducted across each feeling-category and performance level, with feeling intensity as the dependent measure and participants as the predictor. Each of these ANOVAs revealed significant differences between participants' mean intensity values. For all 12 analyses, Levene's Test for homogeneity of variances was violated $(p<.001)$. Based on these violations, post hoc comparisons were made between each participant using Dunnett's C because it accounts for unequal variances (violations of homogeneity), unequal samples, and is more conservative than comparable tests (e.g., Games-Howell). Dunnett's C controls the alpha level more effectively and reduces the chance of type I error (Pallant, 2009). Forty-five post hoc comparisons were made between the 10 players for each of the 12 feeling-category and performance level combinations. In order to reduce likelihood of type I error from so many t tests, a Bonferroni correction for the 45 tests generated a critical alpha level of $<.0011(.05 / 45=.0011)$. No single t test was critical to the study, so results should be interpreted as a general index of idiosyncrasy.

Results are summarized in Table 2, which includes the number of significant $t$ tests at both the .001 and .01 levels. Out of the 540 total post hoc comparisons, 219 significant differences were identified - meaning that $40.6 \%$ of the comparisons of feeling intensity were statistically unique (at the .001 level). At the .01 alpha level, 304 tests (56.3\%) were significant.

Differences in (size) variances of zones. Complementing the comparisons of central tendencies, differences in the width of zones were compared. There is no established statistical procedure to assess whether or not the size of one player's zone is different from another's, so the variance of each zone is proposed to represent the width of individual zones. Accordingly, the variances of intensity ratings were assessed simultaneously for all participants. Twelve such sets of tests were conducted for each feeling-category and performance level. Each player's variance score was divided by the other's, then compared to the critical value from standardized $\mathrm{F}$ tables, factoring in the ( $\mathrm{n}-1)$ number of observations for each player (Lake Tahoe Community College, 2001). Before conducting interindividual comparisons, Levene's test of equality of error variances were assessed to provide an overall appraisal of variance. A summary of Levene's tests and the number of significant comparisons at the .001 and .01 alpha levels are provided in Table 2 . Of the 540 possible comparisons, 220 were statistically different - meaning that $40.7 \%$ of zone widths were unique (at the .001 level). At the .01 level, 289 tests $(53.5 \%)$ were significant.

Summary of individual differences. Sixty-two percent (61.7) of interindividual comparisons across feeling-categories and performance levels showed significant 
differences in central tendency, variance, or both. Good zones displayed the most idiosyncratic tendencies (averaging 67.8\%), followed by average $(62.8 \%$ ) and poor zones (54.4\%). Zones for NF (68.1\%) and PD (67.4\%) feelings had higher differences, compared to ND (61.5\%) and PF (49.6\%) feelings. Thus, players displayed greater idiosyncrasy across good-intensity zones and reversal combinations (PD and NF categories). These trends were consistent at the .01 alpha level, which revealed significant differences in zone size and/or location $76.3 \%$ of the time - surprisingly high given the limited possible range of feeling intensities for zones to distribute across.

\section{Discussion}

The paper provides a large data set of self-reported assessments during changeovers of competitive tennis matches. This is the first study to use probabilistic analysis to develop IZOF profiles with discrete feelings arranged by valence-function categories. The study provides a unique (i.e., pIZOF) approach to the array of methods used to develop idiographic profiles. The advantage of pIZOF profiles is that they use discrete feeling states to develop individualized feeling-performance profiles that are explicitly probabilistic. As such, the items in individual profiles are more meaningful to athletes, and they provide flexibility for researchers to explore either individual discrete feelings, or global categories of items based on function and valence.

Conducting OLR on categories of discrete feeling states yielded pIZOF profiles resembling those published in IAPZ studies. The results provide qualitative, visual, and statistical evidence in favour of the robust finding that feeling-performance relationships are idiosyncratic. Each set of results confirmed that individual differences existed between players, and that these differences were substantive, but also limited. In other words, players were not completely different, but they were more different than identical across their profiles. In spite of the large number of total observations, the numbers of observations for each performance level of each player were occasionally somewhat small. As such, the degrees of freedom available for some variance and post hoc comparisons made the likelihood of significant differences unreliable. Despite this limitation, the current study provides new ways to compare the intensity/location of zones (central tendency), and the size of zones (variance). Stronger statistical methods for generating and describing individual zone profiles - in the form of OLR, $t$ tests, variance comparisons, and other parametric/nonparametric techniques compliment traditional methods of idiographic comparison.

These results expand on the outcomes from Flett (2014, 2015), which are recently published papers from this same data set. Flett (2014) examined the 
accuracy of the pIZOF profiles in terms of their ability to accurately predict, or represent, the relationship between feeling intensity and performance levels. Unique from the data presented in this paper, Flett (2014) used the accuracy data to also compare the effectiveness of the four valence-function categories to four discrete feelings (not aggregated) that were identified by participants as critical triggers for optimal performance. Triggers "were identified by participants as being the most important feelings in their profile - feelings that 'triggered' best or worst performances more so than any other feelings"' (Flett, 2014, p. 413). Triggers were identified by participants as their most important feeling-factors, and were identified through the analysis as the most accurate predictors of tennis performance (more so than the aggregated categories from each participants' profile). It should be noted that in this previous study, both aggregated categories and individual discrete feelings were successfully profiled using the pIZOF method - though limited information about how those profiles were initially developed were provided in that paper - unlike this paper.

In addition to focusing on the accuracy of profiles and the role of discrete triggers in feeling-performance profiling, (Flett, 2014) augments the current paper by providing: descriptions of each participant's profile items (and specific triggers); distributions of performance ratings; a simple method for assessing the accuracy of a feeling-performance profile; and practical recommendations for how simplified profile-based cues can be used by athletes and coaches. The current study augments the earlier publication by offering: more details about how the study was conducted; detailed information about how the pIZOF profiles were developed; and much more descriptive (quantitative and qualitative) data about the idiographic nature of the profiles.

One way that the results from this study did not support previous studies was in the most popular profile items selected by the participants. Based on a sample of 138 athletes from nine sports, Hanin (2000a) identified the most common positive and negative feelings. The 14 rows that received support from at least half of the participants in the current study contained only seven of the 20 most popular items identified by Hanin (2000a, p. 169). The nine sports sampled by Hanin included two racquet sports (squash and badminton), but did not include tennis.

\section{Theoretical and Practical Advantages of Modeling Discrete Feelings}

The current study does not suggest that assessing dimensional affect is inferior to discrete feelings, but that there are advantages to using discrete descriptors of feeling rather than general dimensions. For instance, a single point on an axis that represents dimensional affect can only represent a single 
feeling-experience for that time. Though people can experience more than one feeling at any moment, IAPZ studies have not yet examined the interaction of multiple feeling states at a given moment (e.g., confident yet anxious, or negative but functional, feelings). Furthermore, researchers of the IAPZ framework have not had predictor variables (i.e., arousal, pleasantness, and functionality) interact. This approach fails to differentiate between combinations such as NF and ND states. Consequently, IAPZ researchers typically find that more pleasant and more functional emotions are associated with optimal performance - a potentially misleading finding as it neglects the well-established fact that negative feelings can facilitate performance (Hanin, 2000a; Jones, Hanton, \& Swain, 1994). Valence-function groupings similar to Hanin's could have been developed in previous IAPZ studies (see: Cohen et al., 2006; Edmonds et al., 2006; Golden et al., 2004; Johnson, Edmonds, Moraes, et al., 2007; Johnson, Edmonds, Tenenbaum, \& Kamata, 2007) by coding pleasantness ratings below/above the midpoint as negative or positive; repeating the procedure for functionality ratings; and then combining them. Arousal could then be examined across these feeling interactions.

\section{Other Practical Implications}

In terms of the practical applications for the results, discrete feelings give athletes more personally meaningful descriptors in their profiles. Athletes are more likely to relate to discrete feelings such as confident, strong, nervous, or scared, than to abstract concepts like valence or functionality. Also, as the PNA and Table 1 from Flett (2014, p. 421-422 which depicts the items selected by each participant) show, athletes select a variety of discrete psychobiosocial feelings in their profiles, so reducing a profile to three or four predetermined dimensions of affect does not provide a complete picture of how their personal feelings affect their performance. The idiosyncratic nature of feeling-performance relationships is reinforced by having many feeling descriptors to choose from, rather than limiting athletes to pre-determined nomothetic dimensions of affect. In other words, a completely idiosyncratic and personally meaningful profile cannot be developed unless athletes can select the content of the profile (the feeling items) on their own. If everyone is limited to the same dimensional variables, the profile, by definition, is not idiosyncratic.

Another practical benefit for athletes, coaches, and practitioners is that a probabilistic profile derived from OLR gives practitioners exact information about what the odds are of good, average, or poor performance across feeling intensity. Consider Player 2's pIZOF curves (Figure 2). The PD curves show that while there may be a good-zone from $0-3$, the good-curve is significantly more probable only from 1.5 or lower, and only reaches the critical $50 \%$ level (where 
it is more likely than all other performance outcomes combined) from 0-.75. Practitioners might ask if such a small zone is important, or should we focus on larger, more attainable performance factors than PD feelings? Moreover, for the functional curves (PF and NF), the good-curves are very prominent: there is a $50 \%$ chance (or better) of a good performance from the 6.5 intensity level or higher for both curves. Seeing pIZOF profiles may help athletes to understand how their performance changes across intensity and provides athletes with a visual depiction of how their feelings affect their performance. To support these performance profiles, athletes can develop stronger emotional regulation skills or a mindfulness-acceptance-commitment approach (MAC; Gardner \& Moore, 2006) to psychological skills training (see, Flett, 2014, p. 412 for discussion about pIZOF-MAC strategies).

\section{Limitations and Future Directions}

A practical limitation of the IAPZ and pIZOF methods includes that they require large data sets and unique statistical expertise in order to develop probabilistic profile graphs. This limitation extends beyond the scope and methodology of this study, and applies to any feeling-performance profiling that involves OLR. It is not feasible to obtain data about feeling states during competitions in some sports; and without collecting observations during competition, it would be very difficult to develop a dataset large enough to run OLRs.

One conventional practice in defining the parameters for each intensity zone should be reconsidered. In IAPZ research, zones are defined as the upperand lower-bounds of intensity associated with the most likely outcome. For instance, the ND graph for Player 2 in Figures 2 and 3 depict a good-performance intensity zone from approximately $0-.75$. However, although there is a $35 \%$ of good performance at .75 , the combined probability of average performance is approximately $45 \%$ (10\% for low- and 35\% for high-intensity feelings). As such, at no point across the range of intensity is the likelihood of optimal performance greater than the likelihood of average or poor performance (if the poor or average probabilities include both the high- and low-intensity curves). Separating average- and poor-performance intensity zones into high- and lowintensity groupings has an empirical rationale, but we should not forget that these "separate" curves in a profile ultimately represent the same performance-level.

The PBS model is the framework, or foundation, of the IZOF model (Hanin, 2000a). There are five dimensions in this dynamical systems framework, of which the context and time dimensions have received relatively little attention. The dissertation that this study originates from also explored the context 
dimension but did not find significant differences in profiles between serve and return of serve contexts (comparing within each participant; see, Flett, 2010). A basketball player, for instance, might have very different optimal feelings and zones when playing offense versus defense or shooting free throws. Future studies should also explore the stability of predictive zones across the course of a season or a full annual cycle.

Future research should verify the accuracy of profile predictions for current and future performances. For instance, if a feeling intensity of "7" falls within an optimal zone, this prediction should be compared to the athlete's actual performance rating. This can be done in retrospect for most studies by using the same data used to develop the profile. Although IZOF researchers have occasionally assessed the accuracy of profile's performance predictions, such analyses have not been conducted in IAPZ studies. The accuracy of profiles is an important statistic, and should be provided as often as possible in both lines of idiographic research. An exploratory analysis of profile accuracy using the same data set from this paper indicated that pIZOF profiles accurately predicted performance levels two-thirds of the time (64\%); and participants' top-three favorite triggers (i.e., the discrete feelings that participants identified as being the most critical in the profile) were $70 \%$ accurate (see, Flett, 2014).

\section{Conclusion}

A goal of this study was to remind researchers and practitioners who are interested in developing probabilistic profiles, that such profiles can be developed from discrete feelings rather than abstract dimensional affect, and that probabilistic (OLR-based) methods are compatible with the well-established IZOF model. The results confirmed that the IZOF model can incorporate OLR as a statistical tool to develop probabilistic profiles. Such pIZOF profiles have the advantage of avoiding undefined gaps between zones and overlapping zones that do not provide clear feeling-performance predictions. However, unlike IAPZs, pIZOFs profile discrete feeling-items that can be combined into valence-function categories, or profiled as discrete feelings without aggregation (see, Flett, 2012, 2014). For researchers, there is empirical value in using OLR for performance profiling - whether profiling feelings, emotions, or any other PBS factor. For practitioners, there is practical value in developing profiles using discrete feelings (which may be aggregated into dimensional categories if the athlete, practitioner, or researcher choose to do so).

Golden et al. (2004, p.37) explained: "the purpose of [their] study was to replace Hanin's (2000) concept of affect-performance linkage by deploying a probabilistic method." The notion that IAPZ is a sufficient model to "replace" 
the IZOF framework is overstated. The IAPZ method lacks the variety of procedures for determining individual profile content found in Hanin's model. The IZOF model is also more methodologically flexible, and more extensively researched. The addition of the individual psychological crisis theory (see Tenenbaum et al., 2008), which provides the foundational underpinnings of the IAPZ framework, is not as comprehensive as the PBS framework is to the IZOF model's theoretical foundation. The core of the IAPZ method - its use of OLR - appears to be compatible with the existing framework of the IZOF model. As such, the IZOF model can incorporate significant elements of the IAPZ more so than the IAPZ can replace the IZOF. OLR supplements, but does not supplant, the IZOF framework.

\section{Acknowledgements}

I want to thank the team of participants for their dedication to this study, as well as Katie Griffes, Dan Gould, and Gem Sabolboro for their assistance and support.

\section{References}

Borg, G. (1998). Borg's perceived exertion and pain scales. Champaign, IL: Human Kinetics.

Botterill, C., \& Brown, M. (2002). Emotion and perspective in sport. International Journal of Sport Psychology, 33, 38-60.

Cohen, A. B., Tenebaum, G., \& English, R. W. (2006). Emotions and golf performance: An IZOF-based applied sport psychology case study. Behavior Modification, 30(3), 259-280. doi: 10.1177/0145445503261174

Edmonds, W. A., Mann, D. T. Y., Tenenbaum, G., \& Janelle, C. M. (2006). Analysis of affect-related performance zones: An idiographic method using physiological and introspective data. The Sport Psychologist, 20, 40-57.

Ekkikakis, P., \& Petruzzello, S. J. (2000). Analysis of the affect measurement conundrum in exercise psychology I: Fundamental issues. Psychology of Sport \& Exercise, 1, 71-88. 
Flett, M. R. (2010). Individual feeling states and performance during tennis matches: An integration of the IZOF and LAPZ models. Doctoral Dissertation. Retrieved from http://search.proquest.com/docview/744519372

Flett, M. R. (2012). Integrating the IZOF and IAPZ models to assess individual feeling states and performance during tennis matches. Journal of Exercise, Movement, and Sport: Proceedings from the Canadian Society for Psychomotor Learning and Sport Psychology (SCAPPS) Annual Conference, 44(1). Retrieved from http:/ / jps.library.utoronto.ca/index.php/jems/article/view/24776

Flett, M. R. (2014). Exploring the accuracy and simplicity of idiographic feelingperformance models. Journal of Applied Sport Psychology, 26(4), 409-425. doi: 10.1080/10413200.2014.911220

Flett, M. R. (2015). Is flow related to positive feelings or performance? Path analysis of challenge-skill balance and feelings. Sport Science Review, 24, 5-26. doi: 10.1515/ssr-2015-0006

Gardner, F. L., \& Moore, Z. E. (2006). Clinical sport psychology. Champaign, IL: Human Kinetics.

Golden, A. S., Tenenbaum, G., \& Kamata, A. (2004). Affect-related performance zones: An indiographic method for linking affect to performance. International Journal of Sport \& Exercise Psychology, 2, 24-42.

Goleman, D. (Ed.). (1997). Healing emotions: Conversations with the Dalai Lama on mindfulness, emotions, and health. Boston, MA: Shambhala Publications, Inc.

Hanin, Y. L. (1993). Optimal performance emotions in top athletes. In S. Serpa, J. Alves, V. Ferreira \& A. Paula-Brito (Eds.), Sport psychology: An integrated approach. Proceedings from the VIII World Congress of Sport Psychology (pp. 229-232). Lisbon, Portugal: ISSP.

Hanin, Y. L. (1997). Emotion and athletic performance: Individual zones of optimal functioning model. European Yearbook of Sport Psychology, 1, $29-72$.

Hanin, Y. L. (2000a). Emotions in sport. Champaign, IL: Human Kinetics, Inc.

Hanin, Y. L. (2000b). Individual Zones of Optimal Functioning (IZOF) model: Emotion-performance relationships in sport. In Y. L. Hanin (Ed.), Emotions in sport (pp. 65-90). Champaign, IL: Human Kinetics, Inc. 
Hanin, Y. L. (2003). Performance Related Emotional States in Sport: A Qualitative Analysis. Qualitative Social Research, 4(1). Retrieved from doi: http://nbnresolving.de/urn:nbn:de:0114-fqs030151

Hanin, Y. L. (2007). Emotions in sport: Current issues and perspectives. In G. Tenenbaum \& R. C. Eklund (Eds.), Handbook of sport psychology (3 ed., pp. 3158). Hoboken, NJ: John Wiley \& Sons, Inc.

Hanin, Y. L., \& Syrja, P. (1995). Performance affects in junior ice hockey players: An application of the individual zones of optimal functioning model. The Sport Psychologist, 9, 169-187.

Hanin, Y. L., \& Syrja, P. (1996). Predicted, actual, and recalled affect in olympiclevel soccer players: Ideographic Assessments on Individualized Scales. Journal of Sport and Exercise Psychology, 18(3), 325-335.

Johnson, M. B., Edmonds, W. A., Kamata, A., \& Tenebaum, G. (2009). Determining individual affect-related performance zones (IAPZs): A tutorial. Journal of Clinical Sport Psychology, 3(1), 34-57.

Johnson, M. B., Edmonds, W. A., Moraes, L. C., Medeiros Filho, E. S., \& Tenenbaum, G. (2007). Linking affect and performance of an international level archer incorporating an idiosyncratic probabilistic method. Psychology of Sport \& Exercise, 8, 317-335. doi: 10.1016/j.psychsport.2006.05.004

Johnson, M. B., Edmonds, W. A., Tenenbaum, G., \& Kamata, A. (2007). The relationship between affect and performance in competitive intercollegiate tennis: A dynamic conceptualization and application. Journal of Clinical Sport Psychology, 1, 130-146.

Jones, G., Hanton, S., \& Swain, A. (1994). Intensity and interpretation of anxiety symptoms in elite and non-elite sports performers. Personality \& Individual Differences, 17(5), 657-663.

Kamata, A., Tenenbaum, G., \& Hanin, Y. L. (2002). Individual Zone of Optimal Functioning (IZOF): A Probabilistic conceptualization. Journal of Sport \& Exercise Psychology, 24(2), 189-208.

Lake Tahoe Community College. (2001). Comparing Variances Retrieved February 16, 2010, from http://www.ltcconline.net/greenl/courses/201/regression/ comparingVariances.htm 
Lazarus, R. S. (1991). Emotion and adaptation. New York: Oxford University Press, Inc.

Lazarus, R. S. (2000). How emotions influence performance in competitive sports. The Sport Psychologist, 14, 229-252.

Lazarus, R. S. (2001). Relational meaning and discrete emotions. In K. R. Scherer, A. Schorr \& T. Johnstone (Eds.), Appraisal processes in emotion (pp. 37-67). New York: Oxford University Press.

Medeiros Filho, E. S., Moraes, L. C., \& Tenenbaum, G. (2008). Affective and Physiological States during Archery Competitions: Adopting and Enhancing the Probabilistic Methodology of Individual Affect-Related Performance Zones (IAPZs). Journal of Applied Sport Psychology, 20(4), 441-456. doi: $10.1080 / 10413200802245221$

Pallant, J. (2009). SPSS survival manual (3rd ed.). NY: Open University Press.

Raglin, J. S., \& Hanin, Y. L. (2000). Competitive anxiety. In Y. L. Hanin (Ed.), Emotions in sport (pp. 93-111). Champaign, IL: Human Kinetics, Inc.

Russell, J. A. (1980). A circumplex model of anxiety. Journal of Personality and Social Psychology, 39(1161-1178).

Russell, J. A. (1997). How shall an emotion be called? In R. Plutchik \& H. R. Conte (Eds.), Circumflex models of personality and emotions (pp. 205-222). Washington, D.C.: American Psychology Association.

Russell, J. A., Weiss, A., \& Mendelsohn, G. A. (1989). Affect grid: A single-item scale of pleasure and arousal. Journal of Personality \& SocialPsychology, 57, 493-502.

Syrja, P., \& Hanin, Y. L. (1997). Individualized and group-oriented measures of emotion in sport: A comparative study Annual Congress of the European College of Sports Science. Book of abstracts (pp. 682-684). Copenhagen: University of Copenhagen.

Tenebaum, G., Kamata, A., \& Hanin, Y. L. (2002). The individual zones of optimal functioning. Journal of Sport \& Exercise Psychology, 24, S123.

Tenenbaum, G., Edmonds, W. A., \& Eccles, D. W. (2008). Emotions, coping strategies, and performance: A conceptual framework for defining affect-related performance zones. Military Psychology, 20, S11-S37. doi: $10.1080 / 08995600701804772$ 
Tummavuori, M., \& Hanin, Y. L. (2000). Quantifying emotion intensity on the modified Borg's CR-10 scale. Paper presented at the The 5th Annual Congress of the European College of Sport Science, Jyväskylä, Finland.

Watson, D. (2002). Positive affectivity. In C. R. Snyder \& S. J. Lopez (Eds.), Handbook of positive psychology (pp. 106-119). New York: Oxford University Press.

\begin{abstract}
Marc Ryan FLETT, PhD is currently an assistant professor of Coaching and Teaching Studies at West Virginia University in Morgantown, WV (USA) where he has been for the last four years. He earned his Ph.D. in Sport Psychology from Michigan State University after working for five years as the Mental Trainer and Sport Science Service Coordinator (of the Canada Games Program) for the Sport Medicine Science Council of Saskatchewan and Canada Sports Centre - Saskatchewan. His primary research focus is in the area of positive youth development in sport-in particular, the role of coaches as mentors in PYD, and the role that athletes can have in bullying prevention and school leadership. He has also published several papers exploring the role of emotion in nature-base physical activity promotion, sport performance, and youth development. He publishes regularly in coaching and sport psychology journals and presents at the Canadian Society for Psychomotor Learning and Sport Psychology (SCAPPS) national conference.
\end{abstract}

\title{
Corresponding address:
}

M. Ryan Flett

College of Physical Activity \& Sport Sciences, West Virginia University

PAS 215

PO Box 6116

375 Birch St.

Morgantown, WV 26506

USA

Phone: (304) 293-0844

Email: ryan.flett@mail.wvu.edu 